\title{
Policy options for the radio detectability of Earth
}

\author{
Jacob Haqq-Misra \\ Blue Marble Space Institute of Science \\ 1001 4th Ave Suite 3201, Seattle WA 98154 \\ Email: jacob@bmsis.org. \\ Accepted for publication in Futures.
}

\begin{abstract}
The METI risk problem refers to the uncertain outcome of sending transmissions into space with the intention of messaging to extraterrestrial intelligence (METI). Here, I demonstrate that this uncertainty is undecidable by proving that that the METI risk problem reduces to the halting problem. This implies that any proposed moratorium on METI activities cannot be based solely on the requirement for new information. I discuss three policy resolutions to deal with this risk ambiguity. Precautionary malevolence assumes that contact with ETI is likely to cause net harm to humanity, which remains consistent with the call for a METI moratorium, while assumed benevolence states that METI is likely to yield net benefits to humanity. I also propose a policy of preliminary neutrality, which suggests that humanity should engage in both SETI (searching for extraterrestrial intelligence) and METI until either one achieves its first success.
\end{abstract}

Keywords: SETI; METI; radio transmission; Halting problem, detectability

\section{Introduction: The METI risk problem}

Could sending messages into space be dangerous? A recent exchange between Buchanan (2016) and Vakoch (2016) in the journal Nature Physics highlights the ongoing debate as to whether or not messaging to extraterrestrial intelligence (METI) with electromagnetic waves constitutes an existential risk to humanity. The potential risk associated with METI has led some researchers to consider the possibility of a moratorium on METI activities (Shuch \& Almár, 2007; Billingham \& Bedford, 2011). Other analyses suggest that METI could cause either positive or negative consequences through unknown factors that we cannot influence (Penny 2012), while any attempts at concealing our civilization's leakage radiation would be prohibitively burdensome (Shostak 2013).

I define the METI risk problem as the question: would contact with extraterrestrial intelligence (ETI) cause net benefits or net harm for humanity? Because engaging in METI could possibly attract additional attention from ETI (in addition to existing radio signals, biosignatures, and city lights), this question is also directly relevant for determining the 
value of engaging in METI. Following Haqq-Misra et al. (2013), the value of engaging in METI, $U_{M}$, can be expressed in terms of the probability of detection by ETI, $p_{d}$, and the expected value of the magnitude of ETI response, $\langle M\rangle$, as:

$$
U_{M}=p_{d}\langle M\rangle-C .
$$

In Eq. (11), $C$ represents the total financial costs of engaging in METI, while the term $p_{d}\langle M\rangle$ represents the total value (positive or negative) realized from actually conducting METI. The heart of the METI risk problem is the sign of $\langle M\rangle$; we cannot readily predict whether $\langle M\rangle$ should be positive or negative. This leads to fundamental disagreements regarding whether or not to engage in METI, as we cannot know if contact with ETI would be beneficial $\left(\langle M\rangle \geq 0 \Rightarrow U_{M} \geq 0\right)$ or harmful $\left(\langle M\rangle<0 \Rightarrow U_{M}<0\right)$ to humanity.

This paper demonstrates that the METI risk problem is fundamentally undecidable, which suggests three policy options for how to proceed (or not proceed) with METI. Each of these policy options would determine the long-term detectability of Earth's radio spectrum to any extraterrestrial watchers. Likewise, the same policy options should apply to any extraterrestrial civilizations also facing the METI risk problem, which underscores the connection between METI policy and the search for extraterrestrial intelligence (SETI).

\section{Proof of undecidability}

Can the METI risk problem be resolved? Penny (2012) argues that METI could be beneficial or harmful on an existential level, with no ability to distinguish between this range of options until the actual detection of ETI. This line of reasoning suggests that any policies for METI today, such as a METI moratorium, cannot be based upon the requirement for new information.

In this section, I provide a formal demonstration of the argument advanced by Penny (2012). The purpose of this demonstration is to highlight a key feature of the METI risk debate that is sometimes neglected; namely, that the only information that could resolve the METI risk problem is the actual detection of ETI. In particular, I construct a proof by reduction to show that the METI risk problem is undecidable. (Readers who are convinced of this statement, or find it trivial, can skip to the next section.) A decision problem is undecidable if it is impossible to construct an algorithm (or Turing machine) that can provide a yes or no answer to every question in the domain of the problem. I accomplish this proof by demonstrating that the METI risk problem reduces to a known undecidable problem; namely, the halting problem.

The halting problem is a classic decision problem that can be expressed as follows: given an arbitrary Turing machine $T_{\text {halt }}$, can we determine (with a yes or no answer) if $T_{\text {halt }}$ will halt when initialized with input $w$ ? In other words, the halting problem asks whether or not it is possible to determine in advance if a computer program will halt or continue running forever. The halting problem was proved to be undecidable by Alan Turing in 1936, which implies that any computable function that can be reduced to the halting problem is therefore also undecidable.

To begin the proof, I first assume that there exists a decidable Turing machine $T_{M E T I}$ that answers the METI risk problem when given a set of observations $w$. (Note that $w$ represents any general set of information, including astronomical observations, sociological deductions, and risk analysis. $T_{M E T I}$ represents any possible deductive means of using $w$ to arrive at an answer to the METI risk problem.) I construct $T_{M E T I}$ as a finite-state 
machine with three possible states: $q_{0}$ (initial state, $\langle M\rangle$ is unknown), $q_{1}(\langle M\rangle \geq 0)$, and $q_{2}(\langle M\rangle<0)$. When given input $w, T_{M E T I}$ will either remain at $q_{0}$, transition to state $q_{1}$, or transition to state $q_{2}$. T TMETI answers yes if state $q_{1}$ is reached and answers no if state $q_{2}$ is reached. Likewise, if $T_{M E T I}$ remains in state $q_{0}$, then this implies that input $w$ is insufficient to reach a decision.

I next define a Turing machine $T_{M E T I}^{\prime}$ that takes $w$ and $T_{M E T I}$ as input. I construct $T_{M E T I}^{\prime}$ so that $T_{M E T I}^{\prime}$ will transition to state $q$ and halt if and only if $T_{M E T I}$ enters state $q_{1}$ or $q_{2}$ with input $w$. This implies that $T_{M E T I}^{\prime}$ will halt if and only if $T_{M E T I}$ answers yes or no. I have now reduced the resolution of the METI risk problem $T_{M E T I}$ to the question of whether or not $T_{M E T I}^{\prime}$ will halt with input $w$. This is identical to the halting problem (i.e., determining if $T_{\text {halt }}$ will halt with input $w$ ). However, I assumed at the start of this proof that $T_{M E T I}$ is decidable, but I have reduced $T_{M E T I}$ to the undecidable problem $T_{\text {halt }}$. Therefore, I have arrived at a contradiction and must conclude that $T_{M E T I}$ is undecidable.

\section{Policy options}

If the METI risk problem is undecidable, then how should we as a civilization decide whether or not to engage in METI? No information, prior to the actual discovery of ETI, can provide an unambiguous prediction of benefits or harm from ETI contact. In order to make any progress on this question, we must therefore make assumptions about the terms in Eq. (1D).

\subsection{Precautionary Malevolence}

One approach, adopted by many critics of METI, is that of precautionary malevolence. Lacking any means of deciding the METI risk problem, a policy of precautionary malevolence makes the assumption that $\langle M\rangle<0 \Rightarrow U_{M}<0$, which implies that we should not engage in METI. Arguments in favor of this policy include the recognition that primitive human societies on Earth tend to collapse, at least culturally, when directly contacted by our modern technological civilization (Diamond, 1999). Given the magnitude of possible risks, so the argument goes, we should refrain from engaging in METI, or at least give careful thought to the information content of any messages, in order to avoid increasing any risk from ETI contact to our own civilization (Barkow 2014).

One version of a policy of precautionary malevolence is the proposal to enact a worldwide METI moratorium until the METI risk problem can be resolved (Shuch \& Almár, 2007; Billingham \& Bedford, 2011). However, I have shown above that the METI risk problem is undecidable, so no new information (apart from the actual discovery of ETI) can resolve this issue. Thus, a METI moratorium cannot be justified on the basis of a learningbefore-doing decision principle, because no amount of exploration or research can resolve an undecidable problem. Korbitz (2014) has similarly discussed the limited applicability of the precautionary principle to defend a METI moratorium. The only possible learning that could resolve this ambiguity is if SETI actually succeeds in discovering a signal from ETI - in which case the decision to lift the moratorium would become a matter of debate. Thus, a METI moratorium on the basis of learning-before-doing would effectively prohibit METI activities entirely until SETI first succeeds.

A modified version of precautionary malevolence is the idea that we should wait until we are "more evolved" as a species before we think about engaging in METI. This position presumes that human civilization currently lacks the moral capacity to engage in contact 
with ETI, but future humans (or transhumans) may evolve greater sensibilities than we know now. This version of precautionary malevolence would even suggest that we should refrain from engaging in METI even if SETI succeeds - instead, we should wait until a more distant time in the future when we have evolved as a species. Although I cannot predict the future of human ethics, I will assume that any criterion based upon human evolution must require at least hundreds to thousands of years of civilizational development in order for culture and biology to significantly change from today. If this is the case, and if we assume a policy of precautionary malevolence, then it becomes fair to ask the question: why engage in SETI at all? If we have no plans of replying at all until a distant and unspecified point in the future, then why even bother searching? If human ethics is the limiting factor, then perhaps we should abandon both SETI and METI and focus instead on improving our civilizational values.

\subsection{Assumed Benevolence}

Another approach, adopted by many advocates of METI, is that of assumed benevolence. Lacking any means of deciding the METI risk problem, a policy of assumed benevolence makes the assumption that $\langle M\rangle \geq 0 \Rightarrow U_{M}>0$, which implies that we should consider engaging in METI now. The primary argument in favor of this policy is that any advanced ETI should already have detected our technological civilization, regardless of whether or not we engage in METI. In addition to the radio leakage that has been emanating from Earth for nearly one hundred years (Scheffer, 2004), other tell-tale signs of intelligent life on Earth include our city lights (Loeb \& Turner, 2012) and the spectral signature of our atmosphere (Schneider et al., 2010). Any possible harm that ETI could cause us, so the argument goes, should have already occurred, so we need not worry about any catastrophic threats that could result from METI. Furthermore, the mere discovery of ETI is presumed to be beneficial (if only for scientific and philosophical purposes), which leads toward the conclusion that any success that arises from METI would provide net benefits. Some optimists further imagine that contact with ETI could initiate an interstellar conversation that would bestow us with advanced knowledge (e.g., Sagan 1973) or even welcome us into a galactic club (Bracewell, 1976), bringing about tremendous benefits that would otherwise have been impossible.

A particular version of assumed benevolence examines the possibility of technological asymmetry between us and ETI (Vakoch, 2011). Any ETI that we might contact will invariably be more advanced than us (see, e.g., Baum et al., 2011), which suggests that we might make more widespread use of radio technology than them. By this argument, SETI may never succeed because advanced ETI neither transmit unintentional leakage nor intentional transmissions in our direction. This technological asymmetry suggests that the burden of transmission falls upon the younger, less advanced, civilization. Given that ETI should already know about our existence, our current technological position may place us in an ideal situation to initiate contact with nearby, non-malevolent, ETI.

A policy of assumed benevolence suggests that METI should be beneficial in general, so we should start thinking about actively transmitting for METI as we continue passively exploring through SETI observations. However, assumed benevolence does not necessarily suggest that we should transmit at all costs; METI is expensive, after all, and the costs of engaging in METI must be weighed against the (unknown) magnitude of benefits that would arise from contact with ETI (i.e., does $p_{d}\langle M\rangle \geq C$ in Eq. (1)?). A policy of assumed benevolence is inconsistent with the idea of a METI moratorium, although this policy also does not necessarily imply that we must start transmitting today. For example, the cost 
of METI will decrease significantly in the coming decades when missions such as TESS, PLATO, and JWST begin to characterize nearby exoplanets, which will provide a much better target list for both SETI and METI.

\subsection{Preliminary Neutrality}

A third approach to the METI risk problem is a policy of preliminary neutrality. Lacking any means of deciding the METI risk problem, a policy of preliminary neutrality makes the assumption that $\langle M\rangle=0 \Rightarrow U_{M}=-C$. This policy is takes no position on the likely behavior, social structure, or technological capabilities of ETI. This implies that there is no significant reason to engage in METI, while the only reason to oppose METI is based upon cost limitations. Evidence for preliminary neutrality include the lack of response by ETI to the handful of METI attempts that could have succeeded by now.

The policy of preliminary neutrality implies that the value of METI is simply the cost of transmitting. This policy can be used to argue in favor of prioritizing relevant public resources for other purposes, such as SETI or exoplanet characterization. However, this policy is also consistent with the desires of individuals, private corporations, and wealthy sponsors to engage in METI at their own expense (for whom $C$ is an acceptable sunk cost).

Preliminary neutrality remains inconsistent with the idea of a METI moratorium, although it cannot make a strong argument for the prioritization of METI. Under a policy of preliminary neutrality, activities that could potentially lead to the discovery of ETI- such as any SETI or METI programs, and possibly exoplanet characterization-should continue unabated as funding and enthusiasm permit until the actual discovery of ETI. Once this discovery occurs, all METI programs should be suspended immediately to allow for global discussion of this unprecedented result. Preliminary neutrality recognizes the possibility that speculative endeavors like METI and SETI are probably unlikely to succeed, so there is no reason to prohibit either of these activities until direct evidence shows otherwise.

\section{Conclusion: Implications for detectability}

I have demonstrated that the METI risk problem is undecidable. That is, the question "would contact with ETI cause net benefits or net harm for humanity?" cannot be unambiguously resolved prior to the actual detection of ETI. Based on this proof, three major policy options remain:

1. Precautionary malevolence: contact with ETI is likely to cause net harm to humanity, so we should not engage in METI until SETI succeeds.

2. Assumed benevolence: contact with ETI is likely to yield net benefits to humanity, so we should begin engaging in cost-effective METI along with SETI.

3. Preliminary neutrality: contact with ETI is unlikely to occur, so we may as well continue with SETI and METI until one of them succeeds.

Precautionary malevolence, particularly if it leads to a METI moratorium, would decrease Earth's long-term detectability in order to mitigate potential risk. An extreme form of such a moratorium would consider not just radio transmissions, but also optical evidence of civilization and even the presence of spectral technosignatures in Earth's atmosphere. Such a policy would decrease the likelihood of Earth's detection by ETI astronomers, and 
may also correlate to a decreased probability of success for SETI. Under an active policy of precautionary malevolence, future Earth would be intentionally much less detectable than Earth today.

Assumed benevolence encourages engagement in METI today as a supplement to SETI, which would increase the overall strength of radio (and perhaps optical) transmissions from Earth. Most METI attempts today target individual star systems, particularly those suspected to harbor habitable planets, which can increase Earth's detectability in the direction of transmission. Under a policy of assumed benevolence, future Earth would be intentionally more detectable than Earth today, although such an increase would not be uniform in all directions or across all wavelengths.

Preliminary neutrality acknowledges that SETI and METI are both unlikely to succeed. Human civilization faces a wide range of catastrophic and existential threats, none of which require intervention by ETI, so resources should be prioritized toward ensuring a long-term sustainable future. Under a policy of preliminary neutrality, the detectability of future Earth would remain approximately the same as today. METI, as well as SETI, would remain viable under preliminary neutrality, with scales limited only by the availability of resources. Preliminary neutrality acknowledges that SETI and METI are both extremely

low-probability gambles, but the success of either one would lead to a transformational jackpot.

\section{Acknowledgments}

This research did not receive any specific grant from funding agencies in the public, commercial, or not-for-profit sectors.

\section{References}

Barkow, J. H. (2014). Eliciting Altruism While Avoiding Xenophobia: A Thought Experiment. In Extraterrestrial Altruism (pp. 37-48). Springer, Berlin, Heidelberg.

Baum, S. D., Haqq-Misra, J. D., \& Domagal-Goldman, S. D. (2011). Would contact with extraterrestrials benefit or harm humanity? A scenario analysis. Acta Astronautica, 68(1112), 2114-2129.

Billingham, J., \& Benford, J. (2011). Costs and difficulties of large-scale'messaging', and the need for international debate on potential risks. arXiv:1102.1938,

Bracewell, R. N. (1976). The galactic club: Intelligent life in outer space. San Francisco, WH Freeman and Co.

Buchanan (2016). Searching for trouble? Nature Physics 12: 720.

Diamond, J. (1999). To whom it may concern. New York Times Magazine, 5, 68-71.

Loeb, A., \& Turner, E. L. (2012). Detection technique for artificially illuminated objects in the outer solar system and beyond. Astrobiology, 12(4), 290-294. 
Haqq-Misra, J., Busch, M. W., Som, S. M., \& Baum, S. D. (2013). The benefits and harm of transmitting into space. Space Policy, 29(1), 40-48.

Korbitz, A. (2014). The precautionary principle: Egoism, altruism, and the active SETI debate. In Extraterrestrial Altruism (pp. 111-127). Springer, Berlin, Heidelberg.

Penny, A. (2012). Transmitting (and listening) may be good (or bad). Acta Astronautica, 78, 69-71.

Sagan, C. (1973). Communication with extraterrestrial intelligence (CETI). Communication With Extraterrestrial Intelligence.

Scheffer, L. (2004). Aliens can watch "I Love Lucy." Contact in Context, 2(1), lucy.pdf.

Schneider, J., Léger, A., Fridlund, M., et al. (2010). The far future of exoplanet direct characterization. Astrobiology, 10(1), 121-126.

Shostak, S. (2013). Are transmissions to space dangerous? International Journal of Astrobiology, 12(1), 17-20.

Shuch, H. P., \& Almár, I. (2007). Shouting in the jungle: the SETI transmission debate. Journal of the British Interplanetary Society, 60(4), 142-146.

Vakoch, D. A. (2011). Asymmetry in Active SETI: A case for transmissions from Earth. Acta Astronautica, 68(3-4), 476-488.

Vakoch (2016). In defence of METI. Nature Physics, 12: 890. 